\title{
Direct detection of multiple periods in variable stars
}

\author{
C. Schoenaers, J. Cuypers
}

\begin{abstract}
Royal Observatory of Belgium, Avenue Circulaire 3, B-1180 Brussels, Belgium
\end{abstract}

\begin{abstract}
In some observational data sets of variable stars, multiperiodicity was not always recognized as such, and only became apparent when other observational material became available. In other cases, consecutive prewhitening starting with the obviously most significant frequency could lead to a wrong multifrequency solution. A few examples illustrating this are given. Searching directly for a multifrequency solution can often remedy this. Examples are given and the results of applying this technique to a large set of Hipparcos photometric data are communicated. An overview of the multiple periodicities found in $\beta$ Cephei stars and SPB stars is reported in detail.
\end{abstract}

\section{Introduction}

It has been noted that consecutive prewhitening starting with the obviously most significant frequency can lead to a wrong multifrequency solution. Indeed, by successively prewhitening the two (or more) frequencies found in a LombScargle (from now on LS) periodogram (Scargle 1982), one might overlook the influence the real frequencies can have on each other. This is particularly true for close frequencies, because their peaks can overlap and therefore interfere. In other cases a peak due to noise can be higher than the real frequency peak and therefore be identified as a significant frequency. Sometimes an accepted secondary frequency is only marginally present in the data set, but it can be retrieved in combination with another frequencyor other frequencies: see e.g. the Hipparcos data of the $\beta$ Cep star $\beta$ Cru as presented by Cuypers et al. (2002).

Searching simultaneously for multiple frequencies can remedy these problems. The method we applied is straightforward. If two frequencies are searched for, ALL combinations of trial frequencies are scanned for the best solution in the least squares sense. For three or more frequencies the same principle was used. One can remark that as soon as 4 or more frequencies are looked for, computations can take a long time if the data set is large.

We present examples of simultaneous multiple-frequency searches for an extensive set of Hipparcos photometric data on B stars ( $\beta$ Cep stars and Slowly Pulsating B (SPB) stars, both confirmed and candidate). We focus here on stars for which simultaneous frequency search gave a different solution than successive prewhitening, and present periodograms for the most representative targets.

After performing our systematic direct search for multiperiodicity, we compared our results to accepted solutions found in the literature: it appeared that 

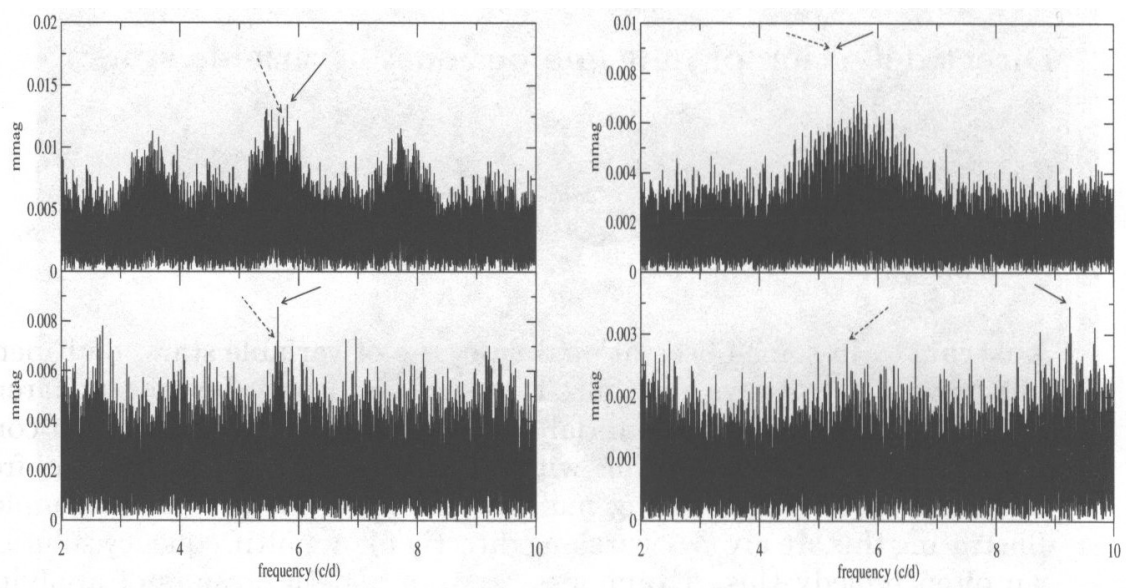

Figure 1. Least squares periodograms for HD 29248 ( $\nu$ Eri) left (top: $3^{\text {rd }}$ frequency, bottom: $4^{\text {th }}$ frequency) and HD111123 $(\beta \mathrm{Cru})$ right (top: $1^{\text {st }}$ frequency, bottom: $2^{\text {nd }}$ frequency). Full arrows point to the highest peaks, dotted arrows to the accepted solution, also found in the Hipparcos data set by searching simultaneously for several frequencies.

even when successive prewhitenings do not yield the accepted solution, simultaneous multiple-frequency search often does. Nevertheless, some discrepancies were noticed, consisting mainly of additional frequencies. A few others are presented in the last section.

Some of the stars we studied were also observed by the Mercator telescope. This new 1.2-m telescope is located on the Roque de los Muchachos observatory on La Palma, Spain. Since 2001 it has been intensively used to observe variable $\mathrm{B}, \mathrm{A}$, and $\mathrm{F}$ main sequence stars. While we present the frequency analysis of the Hipparcos data, we refer to De Cat et al. (these proceedings) for an analysis of the Mercator data.

A more detailed study of the most interesting stars of this survey will be provided in subsequent papers.

\section{2. $\beta$ Cephei stars}

The Hipparcos data set of (candidate and confirmed) $\beta$ Cephei stars we studied consists of 74 stars. We show two examples in Fig. 1: HD 29248 ( $\nu$ Eri) on the left and HD $111123(\beta \mathrm{Cru})$ on the right. In the least squares periodograms of $\nu$ Eri obtained via successive prewhitenings for four frequencies, one sees that for the third and fourth frequencies the highest peaks (full arrows) do not yield the accepted solution (dotted arrows). However, a simultaneous four-frequency search gave as results the accepted frequencies. As for $\beta \mathrm{Cru}$, the second fre- 


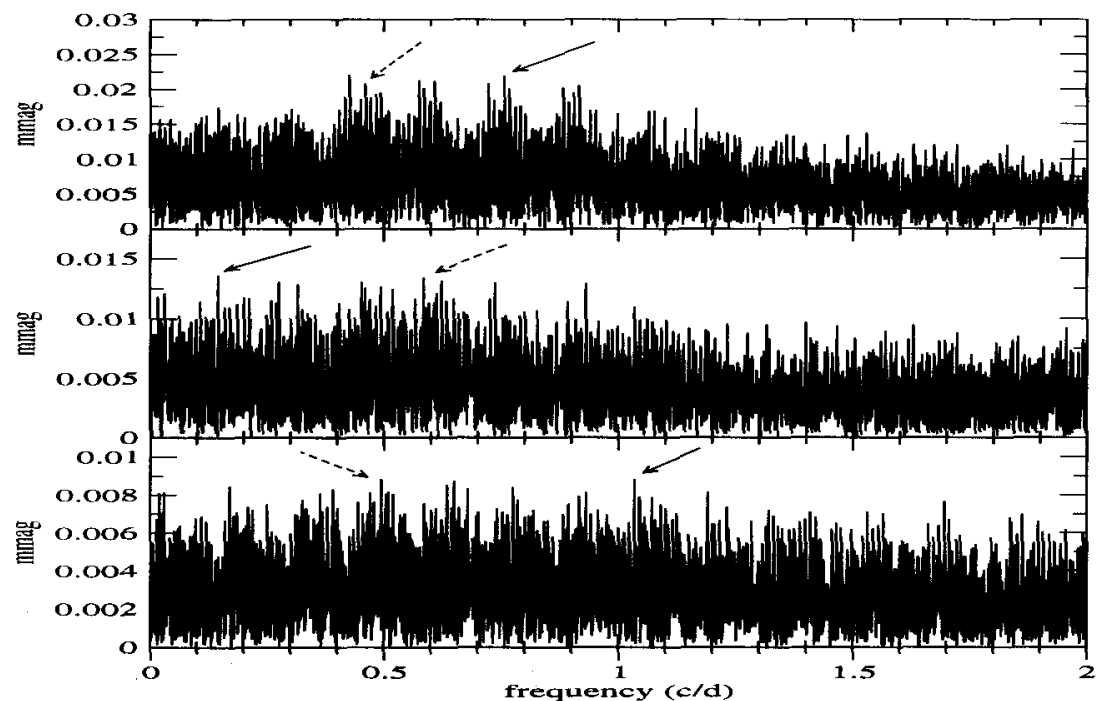

Figure 2. Least squares periodograms for HD 27396. Full arrows point to the highest peaks, dotted arrows to the accepted solution, also found in the Hipparcos data set by searching simultaneously for several frequencies (top: $1^{\text {st }}$ frequency, middle: $2^{\text {nd }}$ frequency, bottom: $3^{\text {rd }}$ frequency).

quency found in Hipparcos using our method is actually the third frequency found in WIRE data; see Cuypers et al. (2002).

\section{3. $\quad$ SPB stars}

The Hipparcos data set of (supposed and confirmed) SPB stars we studied consists of 78 stars. Among these several stars illustrate our point quite vividly. In the least squares periodograms of HD 27396 (HIP 20354, 53 Per) obtained via successive prewhitenings for three frequencies (Fig. 2), one sees that the highest peaks (full arrows) do not yield the accepted solution (dotted arrows). However, a simultaneous two-frequency search gave results much closer to the accepted frequencies, those being yielded by a simultaneous three-frequency search.

\section{New solutions}

Although our main purpose was not to find new pulsation frequencies, but rather to improve the frequency detection method, we compared our results with literature. Besides finding some new pulsation frequencies, we also noticed that 
Table 1. Summary of the modified solutions we obtained. The accepted solutions come from Heynderickx (1992) for $\beta$ Cephei stars, and for SPB stars we compared with $\left({ }^{a}\right)$ De Cat \& Aerts (2002), $\left({ }^{b}\right)$ Huysmans (1998), $\left(^{c}\right)$ Molenda (2000), and $\left({ }^{d}\right)$ Clausen (1996).

\begin{tabular}{lllll}
\hline HD & HIP & Name & Accepted solution & $\begin{array}{l}\text { New solution or } \\
\text { solution with } \\
\text { additional frequency }\end{array}$ \\
\hline 16582 & 12387 & $\delta$ Cet & $\nu_{1}=6.2057 \mathrm{~d}^{-1}$ & $\nu_{2}=5.9072 \mathrm{~d}^{-1}$ \\
64722 & 38438 & V372 Car & $\nu_{1}=9.67067 \mathrm{~d}^{-1}$ & $\nu_{1}=8.6651 \mathrm{~d}^{-1}$ \\
26739 & 19725 & GY Eri & $\nu_{1}=0.7936 \mathrm{~d}^{-1} a$ & $\begin{array}{l}\nu_{2}=0.44393 \mathrm{~d}^{-1} \\
\nu_{3}=0.7249 \mathrm{~d}^{-1}\end{array}$ \\
28475 & 20963 & V1144 Tau & $\nu_{1}=0.67464 \mathrm{~d}^{-1} a$ & $\nu_{2}=0.48431 \mathrm{~d}^{-1}$ \\
64503 & 38455 & QZ Pup & $\nu_{1}=0.89935 \mathrm{~d}^{-1} b$ & $\nu_{1}=1.79848 \mathrm{~d}^{-1}$ \\
& & & $\nu_{2}=1.79847 \mathrm{~d}^{-1} b$ & $\nu_{2}=0.89934 \mathrm{~d}^{-1}$ \\
73654 & 42349 & V363 Vel & $\nu_{1}=0.40717 \mathrm{~d}^{-1} c$ & $\nu_{2}=0.38629 \mathrm{~d}^{-1}$ \\
128585 & 71666 & IS Lup & $\nu_{1}=1.16958 \mathrm{~d}^{-1} c$ & $\nu_{2}=0.72968 \mathrm{~d}^{-1}$ \\
161783 & 87314 & V539 Ara & $\nu_{1}=0.73 \mathrm{~d}^{-1} d$ & \\
& & & $\nu_{2}=0.56 \mathrm{~d}^{-1} d$ & $\nu_{2}=1.56 \mathrm{~d}^{-1}$ \\
\hline \hline
\end{tabular}

some accepted solutions needed to be reconsidered, or that some additional frequencies could be suggested (see Table 1).

Acknowledgments. We acknowledge support from Research Programme G.0178.02 of the Fund for Scientific Research - Flanders (Belgium) This research has made use of the SIMBAD database, operated at CDS, Strasbourg, France.

\section{References}

Clausen, J.V. 1996, A\&A, 308, 151

Cuypers, J., Aerts, C., Buzasi, D., Catanzarite, J., Conrow, T., Laher, R. 2002, A\&A, 392, 599

De Cat, P., Aerts, C. 2002, A\&A, 393, 965

Heynderickx, D. 1992, A\&AS, 96, 207

Huysmans, E. 1998, Undergraduate Thesis, Katholieke Universiteit Leuven, Belgium

Molenda-Żakowicz, J. 2000, in ASP Conf. Ser., Vol. 203, The Impact of LargeScale Surveys on Pulsating Star Research, eds L. Szabados \& D.W. Kurtz (San Francisco: ASP), 434

Scargle, J.D. 1982, ApJ, 263, 835 